\title{
RNA INTERFERENCE
}

\section{Tissue-specific RNA interference in C. elegans}

Watts, J.S. et al. G3 (Bethesda) https://doi.org/10.1534/g3.120.401749 (2020)

RNA interference (RNAi) is widely used to silence gene expression and investigate gene function in living organisms. In Caenorhabditis elegans, RNAi can be induced by feeding animals with bacteria expressing gene-specific double-strand RNA (dsRNA); once ingested by the worms, dsRNA are processed by the RNAi machinery into small interfering RNA (siRNA) that mediate mRNA degradation and gene silencing.

C. elegans strains are available in which a mutation affecting an essential component of the RNAi machinery and resulting in RNAi deficiency can be rescued by a transgene under the control of a tissue-specific promoter. The mutant strain used as a background to generate the tissue-specific RNAi systems needs to be completely RNAi-deficient, so that any RNAi observed is the result of tissue-specific rescue only. However, according to a new study published in $G 3$, some of the $C$. elegans strains deemed 'RNAi deficient' seem to retain substantial RNAi capacity when exposed to RNAi feeding, making them unsuitable for tissue-specific RNAi studies.

The primary objective of the team led by Jennifer Watts from Washington State University was to determine the contribution of different tissues to fatty acid desaturation by feeding dsRNA directed toward fat 1 and fat 4- two genes encoding fatty acid desaturasesto different tissue-specific strains; they would then assess the effects of treatment on the desaturation index using the gas chromatography-mass spectrometry method.

Worms were developed in the background of the RNAi-deficient strain rde-1(ne219) that carries a mutation in the gene encoding RDE-1, the primary

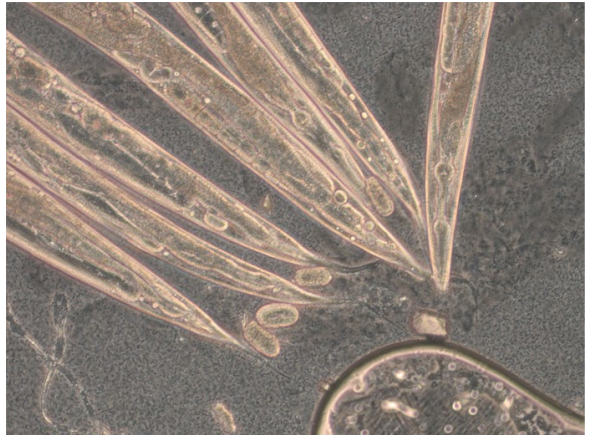

Caenorhabditis elegans and bacteria in culture. Credit: Heiti Paves / Alamy Stock Photo

component of the RNA induced silencing complex (RISC). Although the investigators were expecting to find that fat 1 or fat 4 dsRNA feeding would not affect the fatty acid composition of the strain, they instead observed an important decrease in the desaturation index of the worms, indicating that the strain was not RNAi-deficient.

"Previous reports concluded that rde-1(ne219) was highly resistant to RNAi against several genes when using lethality and fluorescence as indicators. Quantification of metabolite products of targeted enzymes, therefore, provides a more sensitive, quantitative method for assessing the RNAi efficiency of reduction-of-function mutations," write the investigators, who also add that their findings call for caution when interpreting previous tissue-specific RNAi studies using the rde-1(ne219) strain.

Concluding that the rde-1(ne219) strain was not appropriate for their experiments to determine the tissue-specificity of FAT- 1 and FAT-4, the team decided to try a different mutant background. After showing that treatment with fat -1 or fat 4 RNAi did not affect the desaturation index of $r d e-1$ (ne300) worms that carry a different $r d e-1$ mutation, compared with treatment with empty vector, the investigators selected the mutant strain to generate different tissue-specific strains, including transgenic strains with expression of $r d e-1$ gene under the control of intestinal-, epidermal-,germline-, and muscle-specific promoters.

Next, they performed fat 1 and fat-4 RNAi on wild-type, $r d e-1(n e 300)$, and the tissue-specific RNAi strains and showed that RNAi activity in intestinal, epidermal and germline tissues partially reduced the desaturation index, while muscle-specific RNAi showed no detectable change in the desaturation index compared with RNAi performed in rde-1(ne300) worms. Their findings indicate that several tissues but not the muscle contribute to fatty acid desaturation.

The investigators emphasize that the RNAi-specific strains built in the rde-1(ne300) background reported in this study will be useful tools for C. elegans researchers studying a variety of biological processes. Several tissue-specific RNAi strains used in previous studies have been constructed in another background than the $r d e-1$ mutant background, such as sid-1 strains with a mutation in the gene encoding SID-1, a membrane protein that enables the uptake of dsRNA into cells. Here, using their quantitative method relying on the desaturation index, the investigators confirmed that sid-1(qt9) mutants were resistant to RNAi, and are therefore also suitable for future tissue-specific RNAi studies.

Alexandra Le Bras

Published online: 12 October 2020

https://doi.org/10.1038/s41684-020-00667-x 